Pacific

Journal of

Mathematics

STABLE RANK AND REAL RANK OF GRAPH $C^{*}$-ALGEBRAS

J.A. JeOng, G.H. PARK, AND D.Y. Shin 


\title{
STABLE RANK AND REAL RANK OF GRAPH $C^{*}$-ALGEBRAS
}

\author{
J.A. JeOng, G.H. PARK, And D.Y. Shin \\ Dedicated to Professor Sa Ge Lee on his 60th birthday
}

For a row finite directed graph $E$, Kumjian, Pask, and Raeburn proved that there exists a universal $C^{*}$-algebra $C^{*}(E)$ generated by a Cuntz-Krieger $E$-family. In this paper we consider two density problems of invertible elements in graph $C^{*}$-algebras $C^{*}(E)$, and it is proved that $C^{*}(E)$ has stable rank one, that is, the set of all invertible elements is dense in $C^{*}(E)$ (or in its unitization when $C^{*}(E)$ is nonunital) if and only if no loop of $E$ has an exit. We also prove that for a locally finite directed graph $E$ with no sinks if the graph $C^{*}$ algebra $C^{*}(E)$ has real rank zero $\left(R R\left(C^{*}(E)\right)=0\right)$, that is, the set of invertible self-adjoint elements is dense in the set of all self-adjoint elements of $C^{*}(E)$ then $E$ satisfies a condition $(\mathrm{K})$ on loop structure of a graph, and that the converse is also true for $C^{*}(E)$ with finitely many ideals. In particular, for a Cuntz-Krieger algebra $\mathcal{O}_{A}, R R\left(\mathcal{O}_{A}\right)=0$ if and only if $A$ satisfies Cuntz's condition (II).

\section{Introduction.}

Given an $n \times n\{0,1\}$-matrix $A$ with no zero row or column, a family of $n$ partial isometries $S_{i}$ satisfying the relation

$$
S_{i}^{*} S_{i}=\sum_{j=1}^{n} A(i, j) S_{j} S_{j}^{*}
$$

is called a Cuntz-Krieger A-family. In [CK], under a condition (I) on the matrix $A$, it is proved that any two such families generate isomorphic $C^{*}$ algebras, thus the Cuntz-Krieger algebra $\mathcal{O}_{A}$ is well-defined. Furthermore when $A$ satisfies condition (II) which is stronger than (I) the ideal structure of $\mathcal{O}_{A}$ was analysed by Cuntz in $[\mathbf{C}]$.

As a generalization of Cuntz-Krieger algebras one may consider a $C^{*}$ algebra generated by a family of partial isometries satisfying the relation (*) for some infinite $\{0,1\}$-matrix $A$, provided every row of $A$ contains only finitely many 1's, and this has been done in [KPRR] and [KPR] with directed graphs. For any row finite directed graph $E$ with countable vertices 
$\left\{v \mid v \in E^{0}\right\}$ and edges $\left\{e \mid e \in E^{1}\right\}$, the associated graph $C^{*}$-algebra $C^{*}(E)$ is defined to be a universal $C^{*}$-algebra generated by a family of partial isometries $\left\{s_{e} \mid e \in E^{1}\right\}$ and a family of mutually orthogonal projections $\left\{p_{v} \mid v \in E^{0}\right\}$ subject to the relations:

$$
s_{e}^{*} s_{e}=p_{r(e)}, p_{v}=\sum_{s(f)=v} s_{f} s_{f}^{*},
$$

where $r(e)$ (respectively, $s(e))$ denotes the range (respectively, source) vertex of the edge $e$. If $\{A(e, f)\}$ is the edge matrix of $E$ then these relations give a generalized form of $(*)$, that is, $s_{e}^{*} s_{e}=\sum_{s(f)=r(e)} A(e, f) s_{f} s_{f}^{*}$.

If $E$ has no sinks then there is a locally compact $r$-discrete groupoid $\mathcal{G}_{E}$ associated with $E$ whose unit space $\mathcal{G}_{E}{ }^{0}$ is identified with the infinite path space of $E$. Furthermore it is shown in [KPRR], Theorem 4.2 that the groupoid $C^{*}$-algebra $C^{*}\left(\mathcal{G}_{E}\right)$ is isomorphic to $C^{*}(E)$, and hence those useful results on groupoid $C^{*}$-algebras in $[\mathbf{R n} 1]$ and $[\mathbf{R n 2}]$ could be used to analyse the structure of $C^{*}(E)$. One important therem in [KPRR] is about the ideal structure of graph $C^{*}$-algebras; there is an inclusion preserving one-to-one map of saturated hereditary vertex subsets of $E$ into the ideals of $C^{*}(E)$ and moreover if $E$ satisfies a condition $(\mathrm{K})$ then the map is also bijective.

A graph-theoretic condition (L) analogous to Cuntz-Krieger's condition (I) was given in $[\mathbf{K P R}]$, where it was shown that if $E$ is a locally finite directed graph with no sinks and satisfies $(\mathrm{L})$ then a $C^{*}$-algebra generated by a Cuntz-Krieger $E$-family of non-zero elements is isomorphic to $C^{*}(E)$. One interesting result among others in $[\mathbf{K P R}]$ is that $C^{*}(E)$ is $\mathrm{AF}$ if and only if $E$ has no loops. It is also shown in $[\mathbf{D}]$ that every AF-algebra arises as the $C^{*}$-algebra of a locally finite pointed directed graph in the sense of [KPRR]. Recall that every AF algebra $A$ has stable rank one $(\operatorname{sr}(A)=1)$; the set of invertible elements is dense in $A$ (or $\widetilde{A}$ if $A$ is nonunital). In Section 3, we give a necessary and sufficient graph-theoretic condition on $E$ for the graph algebra $C^{*}(E)$ to have stable rank one; $\operatorname{sr}\left(C^{*}(E)\right)=1$ if and only if no loop of $E$ has an exit.

We see from [KPR] that if $E$ is a cofinal graph with no sinks and satisfies $(\mathrm{L})$ then the universal $C^{*}$-algebra $C^{*}(E)$ is simple and it is either $\mathrm{AF}$ or purely infinite. It is also well-known that all AF algebras and purely infinite simple $C^{*}$-algebras have real rank zero, that is, every self-adjoint element can be arbitrarily closely approximated by invertible self-adjoint elements (or in the unitized algebra for a nonunital $C^{*}$-algebra). So it would be interesting to know when a non-simple graph $C^{*}$-algebra can have real rank zero, and we prove in Section 4 that for a locally finite directed graph $E$ with no sinks if the graph algebra $C^{*}(E)$ has real rank zero $\left(R R\left(C^{*}(E)\right)=0\right)$ then the graph must satisfy condition $(\mathrm{K})$. Conversely we also show that for any locally finite graph $E$ with no sinks if $E$ satisfies condition (K) and 
$C^{*}(E)$ has finitely many ideals then $R R\left(C^{*}(E)\right)=0$. In particular, if $E$ is a locally finite graph with no sinks and has finitely many vertices then $R R\left(C^{*}(E)\right)=0$ if and only if $E$ satisfies condition $(\mathrm{K})$. Therefore, for a Cuntz-Krieger algebra $\mathcal{O}_{A}$ associated with a $\{0,1\}$-matrix $A$ satisfying (I), $R R\left(\mathcal{O}_{A}\right)=0$ if and only if $A$ satisfies condition (II) since $A$ can be viewed as a vertex matrix of a finite graph $E$ which has no sinks and satisfies (L) and that the finite graph $E$ satisfies condition $(\mathrm{K})$ is equivalent to that its vertex matrix $A$ satisfies condition (II).

\section{Preliminaries.}

We recall some definitions and notations from $[\mathbf{K P R}]$ and $[\mathbf{K P R R}]$ on directed graphs, graph $C^{*}$-algebras, and groupoids associated with graphs. A directed graph $E=\left(E^{0}, E^{1}, r, s\right)$ consists of countable sets $E^{0}$ of vertices and $E^{1}$ of edges, and the range, source maps $r, s: E^{1} \rightarrow E^{0}$. E is row finite (locally finite) if for each vertex $v \in E^{0}, s^{-1}(v)$ is (both $r^{-1}(v)$ and $s^{-1}(v)$ are) finite. We call a locally finite graph $E$ finite if $E^{0}$ is finite. If $e_{1}, \ldots, e_{n}$ $(n \geq 2)$ are edges with $r\left(e_{i}\right)=s\left(e_{i+1}\right), 1 \leq i \leq n-1$, then we can form a (finite) path $\alpha=\left(e_{1}, \ldots, e_{n}\right)$ of length $|\alpha|=n$, and extend the maps $r, s$ by $r(\alpha)=r\left(e_{n}\right), s(\alpha)=s\left(e_{1}\right)$.

Let $E^{n}$ be the set of all finite paths of length $n$ and

$$
\begin{aligned}
E^{*} & :=\cup_{n \geq 0} E^{n}, r(v)=s(v)=v \text { for } v \in E^{0}, \\
E^{\infty} & :=\left\{\left(\alpha_{i}\right)_{i=1}^{\infty} \mid \alpha_{i} \in E^{1}, r\left(\alpha_{i}\right)=s\left(\alpha_{i+1}\right)\right\} .
\end{aligned}
$$

A vertex $v \in E^{0}$ with $s^{-1}(v)=\emptyset$ is called a sink.

Given a row finite directed graph $E$, a Cuntz-Krieger E-family consists of a set $\left\{P_{v} \mid v \in E^{0}\right\}$ of mutually orthogonal projections and a set $\left\{S_{e} \mid e \in E^{1}\right\}$ of partial isometries satisfying the relations

$$
S_{e}{ }^{*} S_{e}=P_{r(e)}, e \in E^{1} \text {, and } P_{v}=\sum_{s(e)=v} S_{e} S_{e}{ }^{*}, v \in s\left(E^{1}\right) .
$$

From these relations, one can show that every non-zero word in $S_{e}, P_{v}$ and $S_{f}^{*}$ is a partial isometry of the form $S_{\alpha} S_{\beta}^{*}$ for some $\alpha, \beta \in E^{*}$ with $r(\alpha)=r(\beta)$ ([KPR], Lemma 1.1).

Theorem 2.1 ([KPR, Theorem 1.2]). For a row finite directed graph $E=$ $\left(E^{0}, E^{1}\right)$, there exists a $C^{*}$-algebra $C^{*}(E)$ generated by a Cuntz-Krieger $E$ family $\left\{s_{e}, p_{v} \mid v \in E^{0}, e \in E^{1}\right\}$ of non-zero elements such that for any CuntzKrieger E-family $\left\{S_{e}, P_{v} \mid v \in E^{0}, e \in E^{1}\right\}$ of partial isometries acting on a Hilbert space $\mathcal{H}$, there is a representation $\pi: C^{*}(E) \rightarrow B(\mathcal{H})$ such that

$$
\pi\left(s_{e}\right)=S_{e}, \text { and } \pi\left(p_{v}\right)=P_{v}
$$

for all $e \in E^{1}, v \in E^{0}$. 
A finite path $\alpha$ with $|\alpha|>0$ is called a loop at $v$ if $s(\alpha)=r(\alpha)=v$. If the vertices $\left\{r\left(\alpha_{i}\right)|1 \leq i \leq| \alpha \mid\right\}$ are distinct, the loop $\alpha$ is simple.

$E$ is said to satisfy a condition (L) if every loop in $E$ has an exit, and a condition $(\mathrm{K})$ if for any vertex $v$ on a loop there exist at least two distinct loops $\alpha, \beta$ based at $v$, that is, $r(\alpha)=r(\beta)=s(\alpha)=s(\beta)=v, r\left(\alpha_{i}\right) \neq v$ for $1 \leq i<|\alpha|$, and $r\left(\beta_{j}\right) \neq v$ for $1 \leq j<|\beta|$. Note that the condition $(\mathrm{K})$ is stronger than (L) and if $E$ has no loops then the two conditions are trivially satisfied.

If $E$ has no sinks then $E^{\infty} \neq \emptyset$ and we have the following groupoid associated with $E$

$$
\begin{gathered}
\mathcal{G}_{E}=\left\{(x, k, y) \in E^{\infty} \times \mathbb{Z} \times E^{\infty} \mid x_{i}=y_{i+k} \text { for sufficiently large } i\right\} \\
(x, k, y)^{-1}:=(y,-k, x), \\
(x, k, y) \cdot(y, l, z):=(x, k+l, z) .
\end{gathered}
$$

Then the range and source maps $r, s: \mathcal{G}_{E} \rightarrow \mathcal{G}_{E}{ }^{0}$ are given by

$$
r(x, k, y)=x, s(x, k, y)=y .
$$

$\mathcal{G}_{E}$ is a locally compact $r$-discrete groupoid with respect to a suitable topology and $\mathcal{G}_{E}{ }^{0}$ is identified with $E^{\infty}$. Furthermore the groupoid algebra $C^{*}\left(\mathcal{G}_{E}\right)$ is isomorphic to the graph $C^{*}$-algebra $C^{*}(E)$ by Theorem 4.2 of [KPRR].

\section{Stable rank of $C^{*}(E)$.}

Recall that a $C^{*}$-algebra $A$ has stable rank one $(\operatorname{sr}(A)=1)$ if the set $A^{-1}$ of all invertible elements is dense in $A$ (in $\widetilde{A}$ if $A$ is non-unital). One can show that every $C^{*}$-algebra $A$ with $\operatorname{sr}(A)=1$ is stably finite, and so there is no infinite projection in $A$. If two $C^{*}$-algebras $A$ and $B$ are strong Morita equivalent, in particular if they are stably isomorphic, then $\operatorname{sr}(A)=1$ if and only if $\operatorname{sr}(B)=1$ ([BP2], [Rf] $)$.

Lemma 3.1 ([BP2, Proposition 6.4]). Let I be an ideal of a $C^{*}$-algebra $A$. Then $\operatorname{sr}(A)=1$ if and only if $\operatorname{sr}(I)=\operatorname{sr}(A / I)=1$ and every invertible element lifts (that is, $\left.(\widetilde{A} / I)^{-1}=\widetilde{A}^{-1} / I\right)$.

We say that a subgraph $H$ of $E$ has no exit if $e \in E^{1}, s(e) \in H^{0}$ implies $e \in H^{1}$.

Lemma 3.2 ([KPR, Proposition 2.1]). If $H$ is a subgraph of a directed graph $E$ with no exit then

$$
I:=\overline{\operatorname{span}}\left\{s_{\alpha} s_{\beta}{ }^{*} \mid \alpha, \beta \in E^{*}, r(\alpha)=r(\beta) \in H^{0}\right\}
$$

is a closed ideal of $C^{*}(E)$ strong Morita equivalent to the hereditary $C^{*}$ subalgebra $B:=\overline{\operatorname{span}}\left\{s_{\alpha} s_{\beta}^{*} \mid \alpha, \beta \in H^{*}\right\}$. 
We call a vertex $v$ cofinal if for any infinite path $x=\left(x_{1}, x_{2}, \ldots\right) \in E^{\infty}$ there is a finite path $\alpha \in E^{*}$ with $s(\alpha)=v$ and $r(\alpha)=s\left(x_{n}\right)$ for some $n$ ([KPRR $]$ ). A directed graph $E$ is said to be cofinal if every vertex is cofinal.

Theorem 3.3. Let $E=\left(E^{0}, E^{1}, r, s\right)$ be a row finite directed graph. Then $E$ has no loop with an exit if and only if $\operatorname{sr}\left(C^{*}(E)\right)=1$.

Proof. If $E$ has no loops then $C^{*}(E)$ is $\mathrm{AF}$ and so $\operatorname{sr}\left(C^{*}(E)\right)=1$. Assume that $E$ has loops and every loop has no exit. Let $H$ be the subgraph of $E$ consisting of all the loops. Since $H$ has no exit, by Lemma 3.2,

$$
I=\overline{\operatorname{span}}\left\{s_{\beta} s_{\gamma}{ }^{*} \mid \beta, \gamma \in E^{*}, r(\beta)=r(\gamma) \in H^{0}\right\}
$$

is an ideal of $C^{*}(E)$ which is strong Morita equivalent to the hereditary subalgebra $B=\overline{\operatorname{span}}\left\{s_{\beta} s_{\gamma}{ }^{*} \mid \beta, \gamma \in H^{*}\right\}$. Let $\alpha$ be a simple loop in $E$, then $v=s(\alpha)$ is cofinal in the subgraph $H_{\alpha}$ consisting only of $\alpha$, and $H_{\alpha}$ has no sinks. Thus $C^{*}\left(H_{\alpha}\right) \cong C^{*}\left(\mathcal{G}_{H_{\alpha}}\right)$ ([KPRR], Theorem 4.2). Let $N=\left\{x \in H_{\alpha}^{\infty} \mid s(x)=v\right\}$, and $\mathcal{G}_{H_{\alpha} N}{ }^{N}$ be the reduction of $\mathcal{G}_{H_{\alpha}}$ to $N$. Then by [KPRR], Theorem 3.1, $C^{*}\left(\mathcal{G}_{H_{\alpha} N}{ }^{N}\right)$ is isomorphic to the full corner of $C^{*}\left(\mathcal{G}_{H_{\alpha}}\right)$, so they are strong Morita equivalent. Since $N$ consists of only one path, say $x$, and $\mathcal{G}_{H_{\alpha}}{ }^{N}=\{(x, k n, x) \mid k \in \mathbb{Z}\} \cong \mathbb{Z}, C^{*}\left(H_{\alpha}\right)$ is strong Morita equivalent to the group $C^{*}$-algebra $C^{*}(\mathbb{Z}) \cong C(\mathbb{T})$. Since $C(\mathbb{T})$ has stable rank 1 , it follows that $\operatorname{sr}\left(C^{*}\left(H_{\alpha}\right)\right)=1$, and so $\operatorname{sr}\left(B_{\alpha}\right)=1$, where $B_{\alpha}:=\overline{\operatorname{span}}\left\{s_{\beta} s_{\gamma}{ }^{*} \mid \beta, \gamma \in H_{\alpha}^{*}\right\}$, because $B_{\alpha}$ is a quotient algebra of $C^{*}\left(H_{\alpha}\right)$. Thus $\operatorname{sr}\left(I_{\alpha}\right)=\operatorname{sr}\left(B_{\alpha}\right)=1$, where

$$
I_{\alpha}:=\overline{\operatorname{span}}\left\{s_{\beta} s_{\gamma}{ }^{*} \mid \beta, \gamma \in E^{*}, r(\beta)=r(\gamma) \in H_{\alpha}^{0}\right\} .
$$

Therefore $\operatorname{sr}(I)=1$ since $I$ is the direct sum of the ideals $I_{\alpha}$.

Now, let $D$ be the $C^{*}$-subalgebra of $C^{*}(E)$ generated by

$$
\left\{s_{e} \mid e \in E^{1} \backslash H^{1}\right\} \cup\left\{p_{v} \mid v \in E^{0}\right\},
$$

which is a Cuntz-Krieger $G$-family for the subgraph $G=\left(E^{0}, E^{1} \backslash H^{1}\right)$ of $E$. Thus by Theorem 2.1 there is a $*$-homomorphism from $C^{*}(G)$ onto $D$. Since $G$ has no loops at all, $C^{*}(G)$ is an AF algebra having stable rank one, so we have $\operatorname{sr}(D)=1$ by Lemma 3.1 .

It is clear that under the canonical projection $\pi: C^{*}(E) \rightarrow C^{*}(E) / I$ the subalgebra $D$ of $C^{*}(E)$ maps onto $C^{*}(E) / I$ and hence the stable rank of $C^{*}(E) / I$ is one as a homomorphic image of an algebra of stable rank one. Also, every invertible element in the AF algebra $\pi(\widetilde{D})\left(=\widetilde{C^{*}(E) / I}\right)$ is connected to the unit, whence it lifts to an invertible element in $\widetilde{C^{*}(E)}$. Then by Lemma 3.1, $\operatorname{sr}\left(C^{*}(E)\right)=1$.

Conversely, suppose that $E$ has a simple loop $\alpha=\left(\alpha_{1}, \ldots, \alpha_{n}\right)$ with an exit at $v=s(\alpha)$. It is easy to see that the projection $p_{v}$ is infinite, so the algebra $C^{*}(E)$ is not stably finite, whence $\operatorname{sr}\left(C^{*}(E)\right) \neq 1$. 
Lemma 3.4. If $V$ is the set of all sinks in $E$ then

$$
I:=\overline{\operatorname{span}}\left\{s_{\alpha} s_{\beta}^{*} \mid \alpha, \beta \in E^{*}, r(\alpha)=r(\beta)=v \text { for some } v \in V\right\}
$$

is a closed two-sided ideal of $C^{*}(E)$. With $E^{*}(v)=\left\{\alpha \in E^{*} \mid r(\alpha)=v\right\}$, we have

$$
I \cong \oplus_{v \in V} \mathcal{K}\left(\ell^{2}\left(E^{*}(v)\right)\right) .
$$

Proof. For each $v \in V$, let

$$
I_{v}:=\overline{\operatorname{span}}\left\{s_{\alpha} s_{\beta}{ }^{*} \mid \alpha, \beta \in E^{*}, r(\alpha)=r(\beta)=v\right\} .
$$

Then by Corollary 2.2 of $[\mathbf{K P R}], I_{v}$ is a closed ideal of $C^{*}(E)$ and isomorphic to $\mathcal{K}\left(\ell^{2}\left(E^{*}(v)\right)\right)$. If $\beta, \gamma \in E^{*}$, with $r(\beta)=v_{i}, r(\gamma)=v_{j}$, then $s_{\beta}^{*} s_{\gamma}=0$ when $i \neq j$, whence the ideals are mutually orthogonal.

If a (locally finite) directed graph $E$ has sinks then it might not contain any infinite paths so that we can not directly apply results on groupoid $C^{*}$-algebras since the groupoid $\mathcal{G}_{E}$ associated with $E$ was invented to have its unit space consisting of infinite paths in $E$. In case $E$ has no sinks, in $[\mathbf{K P R R}]$, an isomorphism of lattice of saturated hereditary subsets $V$ of $E^{0}$ into the lattice of ideals $I(V)$ in $C^{*}(E)\left(\cong C^{*}\left(\mathcal{G}_{E}\right)\right)$ was established and it is shown that the quotient algebra $C^{*}(E) / I(V)$ is isomorphic to the graph algebra $C^{*}(G)$ for a certain subgraph $G$ of $E$. The proof applies the results on ideal structure of groupoid algebras obtained in [Rn1, Rn2]. See Section 4 for this isomorphism. In the following we show a similar assertion when $V$ is the set of all sinks in $E$. For this, we need to recall that a vertex subset $H$ of $E^{0}$ is saturated if whenever $v \in E^{0}$ emits only edges $e$ with $r(e) \in H$, we have $v \in H$. The smallest saturated vertex subset containing $V$ is called the saturation of $V$.

Theorem 3.5. Let $E=\left(E^{0}, E^{1}, r, s\right)$ be a locally finite directed graph with the set $V$ of sinks. Then there is a subgraph $G=\left(E^{0} \backslash H,\left\{e \in E^{1} \mid r(e) \notin\right.\right.$ $H\})$ of $E$ with no sinks such that $C^{*}(E) / I(V)$ is isomorphic to $C^{*}(G)$, where $H$ is the saturation of $V$ and $I(V)=\overline{\operatorname{span}}\left\{s_{\alpha} s_{\beta}{ }^{*} \mid \alpha, \beta \in E^{*}, r(\alpha)=r(\beta) \in\right.$ $V\}$.

Proof. Note that the ideal $I(=I(V))$ contains the projections $p_{v}$, for $v \in V$. If $e \in E^{1}, r(e)=v$ for some $v \in V$ then $s_{e} \in I$ because $s_{e}=s_{e} s_{e}{ }^{*} s_{e}=$ $s_{e} p_{v} \in I$. For an edge $e \in E^{1}$ with $r(e) \notin V$ we have

$$
s_{e}=s_{e} p_{r(e)}=\sum_{s(f)=r(e)} s_{e} s_{f} s_{f}^{*} p_{r(e)} \in I
$$

whenever the vertex $r(e)$ emits only edges $f$ with $s_{f} \in I$. If $r(e)$ emits an edge $f$ with $s_{f} \notin I$ then $s_{f} s_{f}{ }^{*} \notin I\left(s_{f}=s_{f} s_{f}{ }^{*} s_{f}\right)$. From $s_{e}{ }^{*} s_{e}=p_{r(e)} \geq$ $s_{f} s_{f}^{*} \notin I$, we see that $s_{e}{ }^{*} s_{e} \notin I$, so $s_{e} \notin I$. Thus

$s_{e} \in I \Longleftrightarrow$ either $r(e) \in V$ or $r(e)$ emits only edges $f$ with $s_{f} \in I$. 
Now let $\pi: C^{*}(E) \rightarrow C^{*}(E) / I$ be the canonical surjective homomorphism. Then $\pi\left(C^{*}(E)\right)$ is generated by $\pi\left(s_{f}\right), s_{f} \notin I$. Let $G$ be the subgraph of $E$ obtained from $E$ by deleting the vertices $w$ with $p_{w} \in I$ and edges $f$ with $s_{f} \in I$, that is,

$$
w \in G^{0} \Longleftrightarrow p_{w} \notin I, \quad e \in G^{1} \Longleftrightarrow s_{e} \notin I .
$$

Then $\pi\left(C^{*}(E)\right)$ is generated by $\pi\left(s_{f}\right), f \in G^{1}$. Let $w \in G^{0}$. Then $w \notin V$ and hence $w$ emits edges $e_{1}, \ldots, e_{m}$ in $E$. If $w$ is a sink in $G$ then $s_{e_{i}} \in$ $I, i=1, \ldots, m$, and so $p_{w}=\sum_{i} s_{e_{i}} s_{e_{i}}{ }^{*} \in I$, a contradiciton. Therefore the subgraph $G$ has no sinks.

Let $\pi\left(s_{f}\right) \neq 0$, then $f$ appears in $G$ by $(* *)$. If the vertex $w=r(f)$ emits edges $e_{1}, \ldots, e_{k}, \ldots, e_{m}$ in $E$ such that $s_{e_{1}}, \ldots, s_{e_{k}} \notin I$, and $s_{e_{k+1}}, \ldots, s_{e_{m}} \in$ $I$ then

$$
\begin{aligned}
\pi\left(s_{f}^{*}\right) \pi\left(s_{f}\right) & =\pi\left(\sum_{s(e)=r(f)=w} s_{e} s_{e}^{*}\right) \\
& =\sum_{i=1}^{k} \pi\left(s_{e_{i}}\right) \pi\left(s_{e_{i}}\right)^{*}=\sum_{\substack{s(g)=w=r(f) \\
g \in G^{1}}} \pi\left(s_{g}\right) \pi\left(s_{g}\right)^{*},
\end{aligned}
$$

which means that the partial isometries $\left\{\pi\left(s_{f}\right) \mid f \in G^{1}\right\}$ is a Cuntz-Krieger $G$-family in $\pi\left(C^{*}(E)\right)=C^{*}(E) / I$. Therefore there exists a homomorphism $\phi: C^{*}(G) \rightarrow C^{*}(E) / I$ such that

$$
\phi\left(t_{f}\right)=\pi\left(s_{f}\right), f \in G^{1} \text { and } \phi\left(q_{w}\right)=\pi\left(p_{w}\right), w \in G^{0},
$$

where $\left\{t_{f}, q_{w}\right\}$ is a Cuntz-Krieger $G$-family generating $C^{*}(G)$. On the other hand, one can form a Cuntz-Krieger $E$-family in $C^{*}(G)$ by adding $t_{e}=0$ for $e \in E^{1} \backslash G^{1}$, and $q_{v}=0$ for $v \in E^{0} \backslash G^{0}$ to the family $\left\{t_{f}, q_{w}\right\}$. Then we have a homomorphism $\rho: C^{*}(E) \rightarrow C^{*}(G)$ such that

$$
\rho\left(s_{e}\right)=t_{e}, \rho\left(p_{v}\right)=q_{v}, \quad e \in E^{1}, v \in E^{0} .
$$

Clearly, $I \subset \operatorname{Ker}(\rho)$. Now let $x=\sum \lambda_{\alpha, \beta} s_{\alpha} s_{\beta}^{*} \in \operatorname{Ker}(\rho)$. Then

$$
\pi\left(\sum \lambda_{\alpha, \beta} s_{\alpha} s_{\beta}^{*}\right)=\phi\left(\sum \lambda_{\alpha, \beta} t_{\alpha} t_{\beta}^{*}\right)=\phi \circ \rho(x)=0 .
$$

Thus $x \in \operatorname{Ker}(\pi)=I$. Therefore $\operatorname{Ker}(\rho)=I$ and the map $\rho$ induces an isomorphism from $C^{*}(E) / I$ onto $C^{*}(G)$.

Recall that a $C^{*}$-algebra $A$ is said to be purely infinite if every non-zero hereditary $C^{*}$-subalgebra of $A$ has an infinite projection.

If an $r$-discrete groupoid $\mathcal{G}$ is essentially free and locally contracting then $C^{*}(\mathcal{G})$ is purely infinite $([\mathbf{A}]$, Proposition 2.4$)$. From Lemma 3.4 of $[\mathbf{K P R}]$, we see that the groupoid $\mathcal{G}_{E}$ associated with a locally finite graph $E$ with no sinks is essentially free if and only if $E$ satisfies condition (L). It is also 
known from the same paper that if every vertex connects to a loop with an exit then $\mathcal{G}_{E}$ is locally contracting, so that $C^{*}(E)\left(\cong C^{*}\left(\mathcal{G}_{E}\right)\right)$ is purely infinite. Moreover there is a dichotomy for simple graph $C^{*}$-algebras.

Proposition 3.6 ([KPR, Corollary 3.11]). Let $E$ be a locally finite graph which has no sinks, is cofinal, and satisfies condition $(\mathrm{L})$. Then $C^{*}(E)$ is simple, and

(i) if $E$ has no loops, then $C^{*}(E)$ is $A F$;

(ii) if $E$ has a loop, then $C^{*}(E)$ is purely infinite.

Proposition 3.7. Let $E$ be a locally finite directed graph. If $E$ is cofinal then either $\operatorname{sr}\left(C^{*}(G)\right)=1$ or it is purely infinite simple.

Proof. If $E$ has no loop with an exit then $\operatorname{sr}\left(C^{*}(E)\right)=1$ by Theorem 3.3. Suppose $E$ has a loop with an exit. Since $E$ is cofinal, $E$ can not have a sink. If $E$ has precisely one loop then $E$ satisfies (L) and so $C^{*}(E)$ is purely infinite simple by the previous proposition. Let $E$ have two distinct loops, $\alpha, \beta$. If $\gamma$ is a loop of $E$ then consider the infinite path $x=\alpha \alpha \cdots \alpha=\left(x_{1}, x_{2}, \ldots\right)$ assuming $\gamma \neq \alpha$. Since $E$ is cofinal the vertex $v=s(\gamma)$ connects to $x$ by a finite path, and this shows that the loop $\gamma$ has an exit. Therefore $E$ satisfies $(\mathrm{L})$ and $C^{*}(E)$ is purely infinite simple by Proposition 3.6.

From the proof of the above proposition, we see that for a cofinal graph $E$ with no sinks $C^{*}(E)$ is simple unless $E$ has precisely one loop and the loop has no exit.

\section{Real rank of $C^{*}(E)$.}

Recall that a unital $C^{*}$-algebra $A$ is said to have real rank zero $(R R(A)=$ $0)$ if every self-adjoint element can be arbitrarily closely approximated by invertible self-adjoint elements, that is, $A_{s a}^{-1}$ is dense in $A_{s a}$. For a nonunital $C^{*}$-algebra $A$, we say that $A$ has real rank zero if $\widetilde{A}$ has real rank zero ([BP1]). Then $R R(A)=0$ if and only if $R R(A \otimes \mathcal{K})=0$. Also it is wellknown that $R R(A)=0$ is equivalent to that $A$ satisfies a condition (FS), that is, the set of self-adjoint elements with finite spectra is dense in $A_{s a}$, so $R R(A)=0$ implies that $A$ contains fairly many projections so that the linear span of its projections is dense in $A$. Graph $C^{*}$-algebras $C^{*}(E)$ are basically generated by their partial isometries, and thus they would have plenty of projections and one might expect that most of them have real rank zero. In fact, if $C^{*}(E)$ is simple then it is either AF or purely infinite simple and in both cases it is well-known that these algebras have real rank zero; for real rank of a purely infinite simple $C^{*}$-algebra, see $[\mathbf{Z}]$.

In this section, we first find a necessary condition for a graph $C^{*}$-algebra $C^{*}(E)$ to have real rank zero. We need to review the ideal theory of a graph $C^{*}$-algebra $C^{*}(E)$ for a directed graph $E$ with no sinks. Recall that $C^{*}(E)$ 
can be identified with its infinite path space groupoid model $C^{*}(\mathcal{G})$ and $C^{*}(\mathcal{G}) \cong C_{r}^{*}(\mathcal{G})$ since the groupoid associated with a locally finite directed graph $E$ is amenable ([KPRR], Corollary 5.3). A subset $H$ of the vertex set $E^{0}$ is hereditary if $v \in H$ and $w \in E^{0}$ with $s(\alpha)=v, r(\alpha)=w$ for some $\alpha \in E^{*}$ then $w \in H$.

For a hereditary and saturated vertex set $H \subset E^{0}$, let

$$
U(H)=\left\{x \in E^{\infty} \mid r\left(x_{n}\right) \in H \text { for some } n\right\} .
$$

Then $U(H)$ is an open invariant subset of $E^{\infty}$ (which is identified with the unit space $\mathcal{G}^{0}$ of the groupoid $\mathcal{G}$ associated with the graph $E$ ). The map $H \mapsto$ $U(H)$ is an isomorphism between the lattices of saturated hereditary subsets of $E^{0}$ and open invariant subsets $\mathcal{O}(\mathcal{G})$ of $E^{\infty}$ ([KPRR], Lemma 6.5). On the other hand, for each open invariant subspace $U \subset E^{\infty}\left(=\mathcal{G}^{0}\right)$, the space

$$
C_{c}\left(\mathcal{G}_{U}^{U}\right):=\left\{f \in C_{c}(\mathcal{G}): \operatorname{supp} f \subset \mathcal{G}_{U}^{U}\right\}
$$

is an ideal of $C_{c}(\mathcal{G})$, hence its closure is an ideal $I(U)$ of $C^{*}(\mathcal{G})$. We see from [Rn1], Proposition 4.5 that the correspondence $U \mapsto I(U)$ is a one-to-one order preserving map between $\mathcal{O}(\mathcal{G})$ and the lattice of ideals $\mathcal{J}\left(C^{*}(\mathcal{G})\right.$ ) of $C^{*}(\mathcal{G})$. Thus $H \rightarrow I(U(H))$ is an order preserving isomorphism from the lattice of hereditary saturated vertex subsets into $\mathcal{J}\left(C^{*}(\mathcal{G})\right)$. It is proved in the proof of [KPRR], Theorem 6.6 that the ideals $I(H)$ and $I(U(H))$ coincide, where

$$
I(H):=\overline{\operatorname{span}}\left\{1_{Z(\alpha, \beta)} \mid \alpha, \beta \in E^{*}, r(\alpha)=r(\beta) \in H\right\},
$$

and $1_{Z(\alpha, \beta)}$ is the characteristic function on the compact open subset $Z(\alpha, \beta)$ of the groupoid $\mathcal{G}$.

The isomorphism from $C^{*}(\mathcal{G})$ onto $C^{*}(E)$ obtained in $[\mathbf{K P R R}]$ maps the functions $1_{Z(\alpha, \beta)}\left(\alpha, \beta \in E^{*}, r(\alpha)=r(\beta) \in H\right)$ onto $s_{\alpha} s_{\beta}^{*}$. Therefore we have

$$
I(H)=\overline{\operatorname{span}}\left\{s_{\alpha} s_{\beta}{ }^{*} \mid \alpha, \beta \in E^{*}, r(\alpha)=r(\beta) \in H\right\} .
$$

Furthermore the following is known.

Theorem 4.1 ([KPRR, Theorem 6.6], or [P, Theorem 2.2]). Let $E$ be a locally finite directed graph with no sinks. Then the map $H \mapsto I(H)$ described above is injective, and the quotient algebra $C^{*}(E) / I(H)$ is isomorphic to $C^{*}(F)$ of the directed graph $F:=\left(E^{0} \backslash H,\{e \mid r(e) \notin H\}\right)$. The ideal $I(H)$ is strong Morita equivalent to $C^{*}(K)$ of the directed graph $K:=$ $(H,\{e \mid s(e) \in H\})$. Moreover, if $E$ satisfies the condition $(\mathrm{K})$ then the map $H \mapsto I(H)$ is surjective.

Theorem 4.2 ([BP1]). Let $A$ be a $C^{*}$-algebra and $I$ be an ideal of $A$.

(a) If $R R(A)=0$ then $R R(I)=R R(A / I)=0$.

Suppose $R R(I)=R R(A / I)=0$. Then we have the following. 
(b) $R R(A)=0$ if and only if every projection in $A / I$ lifts to a projection in $A$. In particular if $K_{1}(I)=0$ then every projection lifts.

(c) If $B$ is a $C^{*}$-subalgebra of $A$ with $R R(B)=0$ and $A=B+I$ then $R R(A)=0$.

Now, we can prove our first theorem on real rank of graph $C^{*}$-algebras.

Theorem 4.3. Let $E$ be a locally finite directed graph with no sinks. If $R R\left(C^{*}(E)\right)=0$ then $E$ satisfies condition $(\mathrm{K})$.

Proof. Suppose there is a simple loop $\alpha$ with no exit in $E$. Then the subgraph $H_{\alpha}$ consisiting of $\alpha$ has no exit and generates an ideal $I$ stably isomorphic to $C(\mathbb{T})$, that is, $I \otimes \mathcal{K} \cong C(\mathbb{T}) \otimes \mathcal{K}$, as in the proof of Theorem 3.3. Since $R R(C(\mathbb{T})) \neq 0$ it follows that $R R\left(C^{*}(E)\right) \neq 0$ by Theorem $4.2(\mathrm{a})$, a contradiction, which shows that $E$ satisfies condition (L).

To prove condition $(\mathrm{K})$, let $v$ be a vertex such that there is only one loop at $v$. Let $\beta=\left(\beta_{1}, \beta_{2}, \ldots, \beta_{n}\right)$ be the loop and let $V$ be the set of vertices $w \in V$ such that $w=r(e)$ for an exit $e$ of $\beta$ and $H$ be the smallest hereditary and saturated vertex set containing $V$. Then $V \neq \emptyset$ because $E$ satisfies (L). Moreover, $H$ is a proper subset of $E^{0}$ since vertices on the loop $\beta$ are not elements in $H$. Thus there exists a proper ideal $I(H)$ in $C^{*}(E)$, and the quotient algebra $C^{*}(E) / I(H)$ is isomorphic to $C^{*}(F)$ of the directed graph $F=\left(E^{0} \backslash H,\{e \mid r(e) \notin H\}\right)$. Hence $F$ has a loop $\beta$ with no exit in $F$ and by the argument in the first paragraph of the proof $R R\left(C^{*}(F)\right) \neq 0$. Therefore $R R\left(C^{*}(E)\right) \neq 0$ by Theorem $4.2(\mathrm{a})$.

Corollary 4.4. Let $E$ be a locally finite directed graph with no sinks. If $\operatorname{sr}\left(C^{*}(E)\right)=1$ and $R R\left(C^{*}(E)\right)=0$ then $C^{*}(E)$ is $A F$.

Proof. By Theorem 3.3 and Theorem 4.3, E has no loops, and the assertion follows from Theorem 2.4 in [KPR].

Proposition 4.5. Let $E$ be a locally finite directed graph with no sinks. Then $C^{*}(E)$ is simple if and only if $E$ is cofinal and satisfies $(\mathrm{K})$.

Proof. Suppose $E$ is cofinal and satisfies condition $(\mathrm{K})$ then $C^{*}(E)$ is simple by the proof of [KPRR], Corollary 6.8.

Since the converse has not been proved there in the same proof, we provide one for reader's convenience. To prove the converse, suppose $E$ is not cofinal. Then there exist an infinite path $x$ and a vertex $v$ which cannot connect to $x$ by a finite path. Let $H_{1}$ be the set of all vertices $w$ which can be connected from $v$, that is, there is a finite path $\alpha \in E^{*}$ with $s(\alpha)=v, r(\alpha)=w$. Then $H_{1}$ is the smallest hereditary vertex set containing $v$. Let $H$ be the set of all vertices $w$ satisfying that for any path $\alpha \in E^{*} \cup E^{\infty}$ with $s(\alpha)=w$, if $\alpha \in E^{*}$ then there is another path $\beta \in E^{*}$ such that $s(\beta)=r(\alpha)$ and $r(\beta) \in H_{1}$, if $\alpha \in E^{\infty}$ then $r\left(\alpha_{j}\right) \in H_{1}$ for some $j$. Then clearly $v \in H_{1} \subset H$. We show that $H$ is a saturated hereditary vertex set which does not contain 
vertices on the infinite path $x$. Suppose a vertex $w$ emits edges $e_{1}, \ldots, e_{n}$ and $r\left(e_{i}\right) \in H$ for all $i$. If $\alpha$ is a path with $s(\alpha)=w$ then $\alpha_{1}=e_{j}$ for some $j$ and $\alpha=e_{j} \gamma$ for some path with $s(\gamma)=r\left(e_{j}\right) \in H$. Since $\gamma$ is a path with $s(\gamma)=r\left(e_{j}\right) \in H$, if $\gamma \in E^{*}$ then we can find a path $\beta \in E^{*}$ such that $s(\beta)=r(\gamma)$ and $r(\beta) \in H_{1}$. If $\gamma \in E^{\infty}$ then $r\left(\gamma_{i}\right) \in H_{1}$ for some $i$, and hence $r\left(\alpha_{i+1}\right) \in H_{1}$. Thus $w \in H$, and $H$ is saturated. Now let $u$ be a vertex connected by a finite path $\beta$ from some vertex $w \in H$, that is, $s(\beta)=w, r(\beta)=u$. Then for any path $\alpha$ with $s(\alpha)=u$, the path $\beta \alpha$ starts from $w$, and it is easy to see that $u \in H$, and $H$ is hereditary. Obviously the infinite path $x$ does not meet any vertex in $H_{1}$, hence $H$ is a proper saturated hereditary subset of $E^{0}$. Therefore $C^{*}(E)$ is not simple by Theorem 4.1.

Now suppose $E$ is cofinal but does not satisfy condition $(\mathrm{K})$. Since for a cofinal graph two conditions (K) and (L) are equivalent, $E$ has a loop with no exit. We have already seen from the proof of Theorem 3.3 that such a loop generates an ideal strong Morita equivalent to $C(\mathbb{T})$. Thus $C^{*}(E)$ can not be simple.

We prove the converse of Theorem 4.3 when $C^{*}(E)$ has finitely many ideals.

Theorem 4.6. Let $E$ be a locally finite directed graph with no sinks which satisfies condition $(\mathrm{K})$. If $C^{*}(E)$ has only finitely many ideals then $R R\left(C^{*}(E)\right)=0$. In particular, if $E$ is a finite graph then $R R\left(C^{*}(E)\right)=0$.

Proof. Let $n$ be the number of non-zero ideals in $C^{*}(E)$. We prove our assertion by induction on $n$.

For $n=1, C^{*}(E)$ is simple and $R R\left(C^{*}(E)\right)=0$ since $C^{*}(E)$ is either $\mathrm{AF}$ or purely infinite simple.

Let $n>1$. Let $I(H)$ be a maximal ideal of $C^{*}(E)$ for some hereditary saturated vertex subset $H$ of $E^{0}$. By Theorem 4.1 and induction hypothesis, $I(H)$ and the simple $C^{*}$-algebra $C^{*}(E) / I(H)$ have real rank zero. We show that $C^{*}(E)=I(H)+B$ for some $C^{*}$-subalgebra $B$ isomorphic to $C^{*}(\widetilde{F})$ for a directed subgraph $\widetilde{F}$ (possibly with sinks) of $E$ such that $R R\left(C^{*}(\widetilde{F})\right)=0$ and then apply Theorem 4.2(c). According to Theorem 4.1, $C^{*}(E) / I(H) \cong$ $C^{*}(F)$, where $F=\left(E^{0} \backslash H,\{e \mid r(e) \notin H\}\right)$. Let

$$
V:=\left\{v \in H \mid v=r(e) \text { for some edge } e \in E^{1} \text { with } s(e) \in F^{0}=E^{0} \backslash H\right\} .
$$

If $V=\emptyset$, then $C^{*}(E) \cong I(H) \oplus C^{*}(F)$, and therefore $R R\left(C^{*}(E)\right)=0$ since two direct summands have real rank zero by induction hypothesis. If $V \neq \emptyset$ we set

$$
\widetilde{F}=\left(F^{0} \cup V, F^{1} \cup\left\{f \in E^{1} \mid r(f) \in V, s(f) \in F^{0}\right\}\right) .
$$


Then $V$ is the set of all sinks of $\widetilde{F}$. By Theorem 3.5, $C^{*}(\widetilde{F}) / I(V)$ is isomorphic to the simple $C^{*}$-algebra $C^{*}(F)$, where

$$
I(V)=\overline{\operatorname{span}}\left\{s_{\alpha} s_{\beta}^{*} \mid \alpha, \beta \in \widetilde{F}^{*}, r(\alpha)=r(\beta) \in V\right\} .
$$

Thus $R R\left(C^{*}(\widetilde{F}) / I(V)\right)=R R\left(C^{*}(F)\right)=0$. The ideal

$$
I(V) \cong \oplus_{v \in V} \mathcal{K}\left(\ell^{2}\left(E^{*}(v)\right)\right)
$$

also has real rank zero. Furthermore since $K_{1}(I(V))=0$, by Theorem $4.2(\mathrm{~b})$, $R R\left(C^{*}(\widetilde{F})\right)=0$. Let $B$ be the $C^{*}$-subalgebra of $C^{*}(E)$ generated by the family of nonzero elements $\left\{p_{v}, s_{f} \mid v \in(\widetilde{F})^{0}, f \in(\widetilde{F})^{1}\right\}$. Then this is a Cuntz-Krieger $\widetilde{F}$-family and hence $B$ is a quotient of $C^{*}(\widetilde{F})$. Thus $R R(B)=0$. Now, it is not hard to see that $C^{*}(E)=B+I(H)$, and this completes the proof.

Let $A$ be a $\{0,1\}$-matrix with no zero row or column. Then $A$ can be viewed as a vertex matrix of a finite graph $E$ with no sinks. If $A$ satisfies Cuntz-Krieger's condition (I) in $[\mathbf{C K}]$ then it clearly follows that $E$ satisfies (L) (or, equivalently condition (I) introduced for graphs in [KPR]) from their definitions. By Proposition 4.1 of $[\mathbf{K P R R}]$, the graph algebra $C^{*}(E)$ is also generated by a Cuntz-Krieger $A$-family of partial isometries, hence the Cuntz-Krieger algebra $\mathcal{O}_{A}$ is isomorphic to the graph algebra $C^{*}(E)$. On the other hand, the graph algebra $C^{*}(E)$ is known to be isomorphic to the Cuntz-Krieger algebra $\mathcal{O}_{B}$ associated with the edge matrix $B$ of $E$. Therefore those three algebras are all isomorphic. Furthermore by Theorem 4.3, 4.6, and Lemma 6.1 of [KPRR], we have the following corollary.

Corollary 4.7. Let $A$ be a $\{0,1\}$-matrix with no zero row or column. Suppose A satisfies Cuntz-Krieger's condition (I) and let $E$ be the finite graph having $A$ as its vertex matrix. Then the following are equivalent:

(i) $\operatorname{RR}\left(\mathcal{O}_{A}\right)=0$,

(ii) A satisfies Cuntz's condition (II),

(iii) E satisfies condition (K).

\section{References}

[A] C. Anantharaman-Delaroche, Purely infinite $C^{*}$-algebras arising from dynamical systems, Bull. Soc. Math. France, 125 (1997), 199-225, MR 99i:46051, Zbl 896.46044.

[BP1] L.G. Brown and G.K. Pedersen, $C^{*}$-algebras of real rank zero, J. Funct. Anal., 99 (1991), 131-149, MR 92m:46086, Zbl 776.46026.

[BP2] - On the geometry of the unit ball of a $C^{*}$-algebra, J. Reine Angew. Math., 469 (1995), 113-147, Zbl 834.46041. 
[C] J. Cuntz, A class of $C^{*}$-algebras and topological Markov chains II: Reducible chains and the Ext-functor for $C^{*}$-algebras, Invent. Math., 63 (1981), 25-44, MR 82f:46073b, Zbl 461.46047.

[CK] J. Cuntz and W. Krieger, A class of $C^{*}$-algebras and topological Markov chains, Invent. Math., 56 (1980), 251-268, MR 82f:46073a, Zbl 434.46045.

[D] D. Drinen, Viewing AF-algebras as graph algebras, preprint, 1998.

[KPR] A. Kumjian, D. Pask and I. Raeburn, Cuntz-Krieger algebras of directed graphs, Pacific J. Math., 184 (1998), 161-174, MR 99i:46049, Zbl 917.46056.

[KPRR] A. Kumjian, D. Pask, I. Raeburn and J. Renault, Graphs, groupoids, and Cuntz-Krieger algebras, J. Funct. Anal., 144 (1997), 505-541, MR 98g:46083, Zbl 929.46055.

[P] D. Pask, Cuntz-Krieger algebras associated to directed graphs, Operator Algebras and Quantum Field Theory, Rome, July 1-6, 1996, S. Doplicher, R. Longo, J. Roberts and L. Zsido Eds., International Press, (1997), 85-92, MR 98m:46072, Zbl 921.46050.

[Rn1] J. Renault, A Groupoid Approach to $C^{*}$-Algebras, Lecture Notes in Mathematics, 793, Springer-Verlag, 1980, MR 82h:46075, Zbl 433.46049.

[Rn2] - The ideal structure of groupoid crossed product $C^{*}$-algebras, J. Operator Theory, 25 (1991), 3-36, Zbl 786.46050.

[Rf] M.A. Rieffel, Dimension and stable rank in the K-theory of $C^{*}$-algebras, Proc. London Math. Soc., 46 (1983), 301-333, MR 84g:46085, Zbl 533.46046.

[Z] S. Zhang, A property of purely infinite simple $C^{*}$-algebras, Proc. Amer. Math. Soc., 109 (1990), 717-720, MR 90k:46134, Zbl 673.46038.

Received May 30, 1999 and revised October 6, 1999. The first author was partially supported by GARC-KOSEF, BSRI and the third author was partially supported by BSRI-97-1420.

BK 21, Mathematical Sciences Division

Seoul National University

SEOUL, 151-742

KOREA

E-mail address: jajeong@math.snu.ac.kr

Department of Mathematics

HANSHIN UNIVERSITY

OSAN, 447-791

KorEA

E-mail address: ghpark@hanshin.ac.kr

Department of Mathematics

UNIVERSity OF SEOUL

SeOul, 130-743

KorEA

E-mail address: dyshin@uoscc.uos.ac.kr 